This work is licensed under a Creative Commons Attribution 4.0 International License.

Ovaj rad dostupan je za upotrebu pod licencom Creative Commons Imenovanje 4.0 međunarodna.

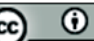

Ksenia KUZMINYKH

UDK 821.09-93

Georg-August-Universität Göttingen, Deutschland

DOI:https://doi.org/10.29162/ANAFORA.v8i2.2

Humboldtallee 19, 37073 Göttingen

ksenia.kuzminykh@uni-goettingen.de

Origineller wissenschaftlicher Beitrag

Original Research Article

Erhalten am 26. 5. 2021

Received: 26 May 2021

Angenommen am 17. 8. 2021

Accepted: 17 August 2021

\title{
DARSTELLUNG DER INNEREN WELTEN VON ERKRANKTEN FIGUREN IN DER DEUTSCHEN KINDER- UND JUGENDLITERATUR
}

\section{Zusammenfassung}

Der Beitrag beschäftigt sich mit der Analyse von Emotionen der erkrankten Figuren und ihrer sozialen Umgebung. Zunächst folgt ein kurzer theoretischer und historischer Überblick. Anschließend werden Emotionsdarstellungen in ausgewählten kinder- und jugendliterarischen Werken analysiert. Ihre Auswahl bemisst sich nach ihrem exemplarischen Charakter für die Explikation von Emotionen der erkrankten Figuren. Das Textkorpus erstreckt sich über einen Zeitraum von ca. 225 Jahren. Ausgehend von der genauen Lektüre der Texte wird ein Muster gebildet, mit dessen Hilfe Emotionsdarstellungen mit typischen Merkmalen typologisiert werden.

Schlïsselwörter: Kinder- und Jugendliteratur, explizite und implizite Emotionsdarstellungen, Krankheitsnarrative 


\section{Forschungsinteresse}

„Wahrscheinlich hat er Angst, sich anzustecken, wenn er mich berührt, Angst, dass ihm dann augenblicklich auch eine Wampe wächst und seinen durchtrainierten Körper entstellt" (Höfler, Tiefseequalle 30) - so beherrscht kann eine an Adipositas leidende Figur auf die fortwährenden Verbalinjurien ihrer Mitschüler reagieren. Andere potenzielle Reaktionen wären ein Suizid aus Desperatheit, eine Metamorphose in eine rachsüchtige Furie oder eine strikte Diät, die sich schleichend in eine Anorexie mit tödlichen Konsequenzen transformieren würde. Die emotionalen Reaktionen der kindlichen und jugendlichen Figuren, die entweder selbst erkranken, oder miterleben müssen, wie jemand aus ihrer näheren sozialen Umgebung von einer unheilbaren Krankheit oder sogar dem Tod aufgesucht wird, variieren. Sie sind so facettenreich wie Krankheitsnarrative des kinder- und jugendliterarischen Diskurses. Daher beschäftigt sich dieser Beitrag mit der Analyse der Emotionsdarstellungen der erkrankten Figuren und ihrer sozialen Umgebung. Der Terminus Krankheit wird als eine psychische oder physische Störung, die an bestimmten Symptomen erkennbar ist, aufgefasst. Emotion wird als ein mentales Phänomen begriffen, das emergente Eigenschaften des physischen Gesamtsystems darstellt, auf einer physiologischen Grundlage beruht, soziokulturell etabliert und sprachlich kodiert ist. Emotionen haben einen Auslöser und richten sich auf ein Objekt. Sie zeichnen sich durch eine temporäre Beschränkung und variierende Intensität aus (Winko 109). In der vorliegenden Studie werden Emotionen als Textphänomene analysiert. Somit grenzt sie sich von der, von Henrike Alfels in ihrer Monografie Literatur und Gefühl vorgeschlagenen, Betrachtungsweise von Emotionen als einem Rezeptionsphänomen, ab. Der Fokus der Typologisierung von Wolf Schmid liegt auf dem fiktiven Bewusstsein der Figuren (Schmid, Mentale Ereignisse 15). In der kognitiven Narratologie ist der Nexus zwischen dem Handeln der Figuren und ihrem Bewusstsein hochrelevant, aber für die vorliegende, an Emotionsdarstellungen orientierte Untersuchung weniger bedeutend. Die Differenzierung in eine explizite Thematisierung und eine implizite Präsentation (Schmid, Mentale Ereignisse 23) findet jedoch Berücksichtigung. Die explizite Thematisierung von Emotionen erfolgt mittels deskriptiver Emotionswörter. Emotionen der Figur werden narratorial (vom Erzähler) oder personal (im mimetischen Figurentext) genannt (Schmid, Elemente der Narratologie 113). Die Indizierung von Emotionen wird mittels des gesamten Spektrums der stilisti-

schen Mittel und symbolischer Ausdrucksformen verwirklicht. Dem Kontext 
kommt dabei eine disambiguierende Bedeutung zu. Diese Mittel dienen ferner als Ausdruck individuell nuancierter emotionaler Erfahrung. Ein Akzent wird auch auf den Konnex zwischen dem Handeln der Figuren und ihren Emotionen sowie ihrer reziproken Motivierung gelegt.

Der kinder- und jugendliterarische Diskurs zum Thema Krankheit zeichnet sich durch die Diversität der Forschungsansätze aus: So beschäftigen sich Iris Schäfer (Schäfer, Von der Hysterie zur Magersucht 19, Schäfer, Zur Ästhetik psychischer Krankheit 11) und Jana Mikota (Mikota 73-90) mit psychischen Erkrankungen. Anne Stemmann betrachtet dissoziative Persönlichkeitsstörung in Anlehnung am Jurij Lotman strukturalistisch als eine Grenzüberschreitung (Stemmann 53). Lisette Gebhard und Michael Klinski gehen auf gesundheitliche Konsequenzen der nuklearen Katastrophen ein (Gebhard/Klinski 9). Marion Rana, Mareike von Müller, Werner Wermeling und Gabrielle von Glasenapp analysieren disability-Narrative unter einer gesellschaftlichen Perspektive (Rana 26, Glasenapp 3, von Müller/Wermeling 35-81). Philipp Schmerheim geht bei dem gleichen thematischen Schwerpunkt komparatistisch vor und fokussiert die Mehrperspektivität der Erzählkonstruktionen (Schmerheim 47). Auch Agnes Blümer, Anika Ullmann (Blümer/Ullmann 58-62) und Sven Nickel (Nickel 381) widmen sich den aktuellen Tendenzen zu Krankheit und Behinderung in Kinder- und Jugendliteratur. Heidi Lexe, Mechthild Blum, Thomas Nesseler, Nicola Mitterer und Werner Wintersteiner richten ihren Blick auf den Tod als Narrativ (Lexe, Wasser 7-9, Lexe, Tod 39-42, Blum/Nesseler 7, Mitterer/ Wintersteiner 152), Anita Schlichter betrachtet illness-narratives als Auslöser für den Kampf um Autonomie und Eigenverantwortung (Schlichter 226). Franziska Pitschke und Simone Weiss beschäftigen sich mit den Krebs-, Aids- und Pest-Narrativen, in denen kindliche und jugendliche Figuren als Helden konzipiert werden (Pitschke 240, Weiss 212), Anne Rüggemeier analysiert graphic illness (Rüggemeier 70-91), während Susanne Balmer die Krankheit in literarischen Weiblichkeitsentwürfen fokussiert (Balmer 45-62). Dieser Überblick zeichnet sich zwar durch einen exemplarischen Charakter aus, aber er macht evident, dass das Thema Emotionen in interpretatorischen Ansätzen nicht im Fokus steht. Emotionen bilden jedoch eine feste, mehr oder weniger deutlich ausgeprägte Konstituente nahezu jedes kinder- und jugendliterarischen illness-narratives. Die vorliegende Studie versucht, durch die Konzentration auf Emotionskodes der narrativen Texte für die ausgewiesene Zielgruppe dieses Desiderat zu schließen. 
Konzeptionell ist der Beitrag wie folgt strukturiert: Nach einem theoretischen und historischen Überblick folgt die Analyse von Emotionen in Krankheitsnarrativen. Unter Rückgriff auf das close-reading-Verfahren sowie auf stilistisch-rhetorische Beschreibungskategorien und ihre Kombination mit emotionstheoretischen Begriffen werden Darstellungen der inneren Welt von Figuren analysiert und in Anlehnung an die von Simone Winko vorgeschlagene Klassifikation in Gruppen eingeordnet (Winko 311). Winkos Typologie orientiert sich zwar an lyrischen Texten, aber sie lässt sich auch für die Analyse von Emotionsdarstellungen in kinder- und jugendliterarischen Narrationen anwenden. Mit ihrer Hilfe können ästhetische Verfahren der Emotionsgestaltung erfasst und präziser analysiert werden.

\section{Historischer Überblick}

Seit der Entstehung der Kinder- und Jugendliteratur vollzog sich ein Paradigmenwechsel bei der Darstellung von Krankheiten und dementsprechend von Emotionen der erkrankten Figuren. Im 18. und 19. Jahrhundert wurde der seit der Antike bekannte Diskurs, in dem Krankheit als Strafe auf eine metaphysische Verfehlung fungierte, in kinder- und jugendliterarische Werke aufgenommen. Die Kranken wurden als stigmatisierte Außenseiter einer gesunden Gesellschaft stilisiert und isoliert (Nickel 382). Die binäre Schwaz-Weiß-Perspektive dominierte die Denkmuster. Der strenge moralisch-erzieherische Impetus kam deutlich zum Vorschein. Die Darstellung von seelischen oder körperlichen Leiden, die aus medizinischer Perspektive nicht näher spezifiziert wurden, erfolgte überzeichnet. Eine selbstverschuldete Erkrankung schloss sowohl eine mitfühlende Reaktion der sozialen Umgebung als auch eine Therapie aus. Erst später wurde bei der Figur des Zappel-Philipps das AD(h)S diagnostiziert (Lüssi 397). Auch andere Figuren aus dem berühmten Band von Heinrich Hoffmanns Struwwelpeter wurden durch medizinische Diagnosen entlastet und vom delinquenten Nonkonformismus freigesprochen.

Ende des 19. Jahrhunderts fand eine Akzentverschiebung statt. Die Tendenz zur admirabilisierenden Idealisierung der resignierten Leidenden und die anvisierte Hinlenkung zur Religiosität dominierten die Narrationen (Norden, Spyri, Felsensprung, Gritlis Kinder, Heidi, Lehrjahre).

Spyris prominenteste Figuren - die an einer schweren Depression in der Groß-

stadt erkrankte Heidi und die in „dem bequemen Rollstuhl sitzende“, verwöhn- 
te Klara (Spyri 72) - genesen dank ihrer Verinnerlichung religiöser Werte. Sie werden mittels einer Metabole an die pietistischen Implikationen der Autorin in einem Konversionsnarrativ angepasst (Kuzminykh, New trends 533). Krankheiten als positiv evaluierte „Lehrjahre der Gemüthsbildung und der Lebenskunst“ (Novalis 686) dienen der „Automythologisierung“ (Frank 123) und werden in einem quest narrative mit einem Zugewinn an Erkenntnis und Reife verknüpft.

Im 20. Jahrhundert vollzieht sich eine Entwicklung von einem präventiven, stark moralisierenden, repressiven Erziehungsstil über den literarischen Tabubruch und Dystopie bis hin zum Inklusionsgedanken (bspw. Ury, Nassauer, Pausewang, Schewenborn, Pausewang, Wolke, Welsh, Hegemann, von der Grün, Höfler, Tiefseequalle, Höfler, Vogel). Die Erkrankungen werden werkimmanent diagnostiziert oder in Paratexten forciert (Rummel/Grütter, Künzler-Behnke, Best, Schäfer, Shreve, Schulz, Tuckermann, Huber). Auch im Bereich der fantastischen quest narratives haben die Figuren oft mit Erkrankungen zu kämpfen (Funke, Reckless, Moers, Richter-Peills). Allerdings lässt sich eine Diskrepanz zwischen dem medizinischen und dem literarischen Diskurs feststellen. Psychische Erkrankungen werden kunstvoll als ein Implikat der Diegese dargestellt. So verhält es sich im Fall von $\mathrm{AD}(\mathrm{h}) \mathrm{S}$. Diese Diagnose wird dazu verwendet, eine herausragende intellektuelle und künstlerische Begabung mit syndromtypischen Ressourcen zu erklären und bemerkenswerte Talente auf $\mathrm{AD}(\mathrm{h}) \mathrm{S}$ zurückzuführen (Best 26, Höfler, Tiefseequalle 163, Höfler, Vogel 29). Es wird suggeriert, dass die idealisierten Aspekte von $\mathrm{AD}(\mathrm{h}) \mathrm{S}$ durch die Einnahme von Medikamenten eliminiert werden. Daher gilt es, die Medikation zu vermeiden (Tuckermann). Darüber hinaus erfolgt eine Rekurrenz auf die axiologische Vorstellung, der zufolge der leichtere Weg der falsche ist. Allein durch die Modifikation der eigenen Persönlichkeit ist die Rekonvaleszenz zu erreichen. Die genetischen Dispositionen, die dieses Syndrom verursachen können, werden ignoriert (Jehle 17).

Ambivalenzkonflikte existieren auch im Fall von literarischen Darstellungen der Anorexie. Das disziplinarische Hungern bzw. die Nahrungsverweigerung galt im ausgehenden 19. Jahrhundert als ein begleitendes Indiz der Hysterie (Hoffmann, Suppen-Kasper). Erst die intensive Beschäftigung mit neurobiologischen und psychogenen Ursachen im vergangenen Jahrhundert führte zur eigenständigen Pathologisierung der anorexia nervosa (Gull 516-17). Das gestörte Selbstbild der an Anorexie Erkrankten und ihr Streben nach Selbstdetermination über den eigenen Körper dienen als Ausgangspunkte für die Krankheitsnarrative dieser Art (Henning von Lange, Stützsack). In Opposition dazu 
stehen literarische Werke, die Adipositas thematisieren. Auch im Fall dieser Erkrankung wird in der Diegese eine axiologische Wertvorstellung etabliert (Höfler, Tiefseequalle 96-110, Freytag 47, Buschendorf, Pressler, Bitterschokolade).

Parallel dazu lässt sich eine kontrovers diskutierte Entwicklung feststellen, die unter dem Begriff Teen Sick Lit (Passante Elman 110) bekannt geworden ist. Werke dieser Richtung legen ihren Fokus auf chronische und unheilbare Erkrankungen. Allerdings werden diese als austauschbar dargestellt und instrumentalisiert, um die Brisanz der beschriebenen Adoleszenzphase sowie der Liebesbeziehung nach dem Beth-March-Syndrom (Passante Elman 110) oder eine metaphysisch-normative Läuterung nach dem Heidi-Syndrom (Reese 146) zu verstärken.

Einen zunehmend wichtigen Aspekt in kinder- und jugendliterarischen Krankheitsnarrativen bilden die Organ- und Blutspende sowie die Organtransplantation, die mit existentialphilosophischen und religiösen Aspekten kombiniert werden (de Velasco, Kein Teil der Welt, Huber).

Mit der Diversität von illness narratives korreliert der Facettenreichtum der Emotionen der Figuren.

\section{Emotionsdarstellung}

In den Krankheitsnarrativen der Kinder- und Jugendliteratur wird das gesamte Emotionsspektrum dargestellt. An der Spitze der Skala stehen verschiedene Varianten von elementaren Emotionen (Angst, Traurigkeit, Freude, Wut, Ekel), die durch komplexe, aus Dyaden bzw. Triaden bestehende Primäremotionen (Liebe, Stolz, Scham) komplettiert werden. Es können sowohl stilistisch-rhetorische Formeln der Emotionsdarstellung als auch Modelle einzelner Emotionen, auf die mit diesen Formeln Bezug genommen wird, variiert werden. Diese beiden Größen sollen als Bezugspunkt für eine Systematisierung des Korpus dienen. Unter dieser Perspektive lassen sich in Anlehnung an Simone Winko folgende Typen differenzieren.

\subsection{Hochkonventionalisierte Metaphern.}

Zum ersten Typus zählen Katachresen (Lakoff/Johnson 150, Winko 334). Prototypische Situationen, in denen Emotionen ausgelöst oder ausgedrückt werden können, komplettieren diese Gruppe. Die Formeln der Emotionsver- 
mittlung variieren durch individuelle Nuancen, aber sie bleiben deutlich erkennbar. Intertextuelle Bezugnahmen mit dem Ziel der Emotionspräsentation verstärken die Wirkung.

Als ein Beispiel für diesen Typus können die Emotionen der an $\mathrm{AD}(\mathrm{h}) \mathrm{S}$ erkrankten Figuren fungieren. Dieses Syndrom wird mit Topoi aus dem Bereich der desaströsen Naturphänomene beschrieben (Höfler, Tiefseequalle 18, Buchholz). Darauf folgen animalische Metaphern (Höfler, Tiefseequalle 163) oder Vergleiche mit Spielzeug (Höfler, Tiefseequalle 137). Die Emotionen der Figuren, bei denen diese Erkrankung diagnostiziert wurde, werden evident: An einem Pol des emotionalen Kontinuums lässt sich die Emotion der Traurigkeit über die defizitäre Konzentrationsfähigkeit, über die leichte Reizbarkeit, über den unbezwingbaren Bewegungsdrang sowie über das sich selbst eingeredete substanzielle charakterliche Defizit (Buchholz, Tuckermann, Schulz) positionieren. Am anderen Pol ist die übertriebene Reaktion der Freude über eben diese Eigenschaften zu finden (Höfler, Tiefseequalle 18, 86).

Als ein weiteres Beispiel ist die Emotionsdarstellung der an Krebs leidenden Figuren zu nennen. Hierfür werden Metaphern verwendet, mit denen innere Welten von Kriegern dargestellt werden: Wut und Hass. Die Erkrankung selbst wird mit einem Kampf oder einem Bürgerkrieg gleichgesetzt (Huber 14, 25).

In diese Gruppe ist auch das stilistische Verfahren einzuordnen, zu dem Lara Schützsack greift. Sie lässt ihre autodiegetische Erzählerin Malina die Krankheit in ihrer unaufhaltsamen, zerstörerischen Entwicklung mit wildem, unkontrollierbar wucherndem Efeu vergleichen. Seine, seit der Antike etablierte, positive Konnotation wird durch die Akzentuierung der hochkonventionalisierten, negativen religiösen und literarischen Symbolik der Gefangenschaft, des Erstickens, der Laszivität und der hierarchischen Dependenz (Galling 153, Butzer 82, Frisch 91, Pope) dominiert. Hinzu kommt die konventionalisierte pseudowissenschaftliche Betrachtungsweise der Schlingpflanze als eines, Bäume und Bauwerke zerstörenden, Parasiten. Der metaphorische Vergleich der Anorexie mit Efeu als einer Verderben bringenden Freundin korreliert mit den Vorstellungen der Betroffenen und ist im medizinischen Diskurs konsensfähig (Bruch 90). Malina indiziert durch diese Metaphorisierung Angst in Bezug auf die $\mathrm{Zu}$ kunft der geliebten Schwester und ihre eigene Zukunft ohne Lucinda (Schützsack 15). Sukzessive verbreitet sich die Kletterpflanze, ergreift das alte schöne Haus, „dringt in sein Gewebe“ ein (Schützsack 20) und verwandelt es in einen 
verwunschenen Ort, so wie Lucindas Krankheit täglich voranschreitet, ihren zierlichen Körper transformiert und ihr die Lebensenergie entzieht (Schützsack 21, 23). Die Wahl dieser romantischen Pflanzen-Metapher expliziert darüber hinaus eine gestörte Selbstwahrnehmung der erkrankten Lucinda. Sie empfindet ihre desaströse Metamorphose als ästhetischen Prozess (Schützsack 131). Der Efeu ruft darüber hinaus allusiv eine andere dahinsiechende Figur in Erinnerung, nämlich Fontanes Effi Briest (Fontane 5). Die enge Verbindung zwischen dem Körper und dem Haus wird mittels weiterer Metaphern verstärkt. Hierzu zählen: Lucindas Tränen und die Tränen des Hauses (Schützsack 26). In diesen Metaphern lässt sich eine Allusion auf die, seit der Romantik etablierte, Parallele zwischen dem Verfall von architektonischen Bauten und dem sozialen bzw. individuellen Niedergang erkennen.

Die Katachrese des goldenen Käfigs, mit der Anorexie (Schützsack 131), aber auch Adipositas (Höfler, Tiefseequalle 165) versinnbildlicht werden, ist im psychiatrischen Fachdiskurs konsensfähig (Bruch, Funke Psychoanalyse). Die an Anorexie Erkrankten möchten sich paradoxerweise mittels der Erkrankung (Bruch 90) aus dem imaginären Käfig befreien. Sie erreichen aber das genaue Gegenteil. Dabei empfinden sie kontinuierlich Schuldgefühle, die mit der Nahrungsaufnahme einhergehen. Der selbstzerstörerische Umgang mit dem eigenen Körper gründet auf dem infantilen Denkstil der an Anorexie erkrankten Personen. Sie geben ihrem Körper an allem Unbehagen und an allen wirklichen oder eingebildeten Mängeln Schuld (Bruch 90). Das wird an den Figuren Lucindas (Schützsack) und Lelles (Henning von Lange) überzeugend demonstriert.

Schließlich ist die Katachrese der Krankheit als einer Abwärtsspirale zu fokussieren. Die Figur Lucindas verschwindet im Keller, in dem sie zunächst ihre erotischen Fantasien auslebt (Schützsack 22-24, 80, 101), der sich jedoch sukzessive zu einem Abgrund der Einsamkeit transformiert. Dieser Eskapismus korreliert mit dem rigorosen Schließen der Ich-Grenze (Funke, Psychoanalyse 87). Lelle schließt die Tür ihres Zimmers, wodurch zwar keine Abwärtsentwicklung, aber ebenfalls das Schließen der Grenze zum Eigenraum (Funke, Psychoanalyse 127) symbolisiert wird.

Die Katachresen werden auch für die Ästhetisierung des Suizids eingesetzt. Als Beispiele sind die Bezeichnung der Freitod sowie der Euphemismus ,Freund Hein` zu nennen. Die Darstellung der Selbsttötung ist einerseits mit Tabus be- 
legt, die mit spezifischen Implikationen zu Religion, Moral, Philosophie und Pädagogik korrespondieren, andererseits übt das Thema eine vermehrte Faszination aus (Herbert, Ehlenberger, Ewers, Blum/Nesseler, Mitterer/Wintersteiner). Hinzu kommt der historische und soziokulturelle Paradigmenwechsel. Am Anfang des 20. Jahrhunderts reduzierte sich die Stigmatisierung des Suizids seitens der Gesellschaft und besonders seitens der Kirche. Damit stand jedem eine freie Entscheidung über den eigenen Tod zu. Emil Stauß verweist kataphorisch im Titel seines Romans Freund Hein auf diese fatale Entwicklung hin. Die Lebensgeschichte der talentierten Zentralfigur Heinrichs, deren Name auf die historische Katachrese verweist, ist teleologisch auf den Suizid ausgerichtet. Der Tod wird als ein loyaler Verbündeter ästhetisiert. Die Entscheidung zur Selbsttötung wird als eine begrüßenswerte Entwicklung dargestellt, die Zweifel an der eigenen Existenz aufhebt. Heiner macht nach dem Schuss in seinen Kopf einen Schritt nach vorn (Strauß 211), wodurch ein Kontrast mit der, in seinem jungen Leben herrschenden, Stagnation akzentuiert wird. Die Selbsttötung wird narratorial als Teil eines göttlichen Plans stilisiert (Strauß 202). Eine ähnliche Konnotation des Selbstmordes findet sich in dem Werk Unterm Rad (Hesse). Die Figur Hans kann dem Leistungsdruck des Wilhelminischen Schulsystems nicht gerecht werden, erkrankt an Depression und begeht Suizid. Auch der musikalisch begabte Christoph erstickt unter der pragmatischen Pedanterie seiner sozialen und familialen Umgebung. Die leidvolle Existenz des Jugendlichen endet tragisch, wenngleich eine Ambivalenz im Hinblick auf seinen Tod bestehen bleibt (Korschunow). Dem Beispiel seiner literarischen Vorgänger folgen Abel Tannatek (Michaelis) und scheinbar Miriam (Paluch/Habeck).

Tobias Elsäßer lässt seine an Depression leidenden Figuren die existenziell philosophische Frage nach dem Sinn des Lebens reflektieren. Die Notwendigkeit, das Leben unter allen Umständen erhalten zu müssen, wird von den Erkrankten verworfen. Marie, die unverschuldet Opfer eines sexuellen Missbrauchs unter der Einwirkung von narkotisierenden Mitteln geworden war, und Nidal, der mit der eignen Inakzeptanz seiner Identität ringt, sehen den kollektiven Selbstmord als einzigen Ausweg aus diesen problembehafteten Situationen (Elsäßer 91, 151). Nidal kommt sich wie in einem Gefängnis vor, in dem er „sogar seinen Gedanken verbietet [...] zu entfliehen“, er fängt sie ein, bevor sie zu mächtig werden und die Kontrolle über ihn übernehmen (Elsäßer 12). Der homosexuelle Jugendliche empfindet eine brennende Ungerechtigkeit, denn schließlich habe man ihn „ohne Prozess, ohne Anwalt, ohne Zeugen“ verurteilt 
(Elsäßer 13) und „er will fliegen. Auf und davon“ (Elsäßer 13). Die pathologische Selbstbestimmung erhält somit für diese Figuren eine psychotherapeutische Nuance: Suizid stellt ein Heilmittel gegen ein psychisches Trauma dar und wird als Inbegriff von Freiheit überhöht (Elsäßer 12, 15, 41, 61, 143).

Die Pathologisierung des Suizids als Symptom einer psychischen Erkrankung, in der Regel der Depression, erfolgt erst nach dem zweiten Weltkrieg (Ehlenberger, Fenner). Diese neue Perspektivierung befreit einerseits die Suizidenten von den Vorwürfen, egozentrisch zu handeln oder feige, schwach, inkompatibel und anpassungsunwillig zu sein. Auf der anderen Seite wird den Suizidenten durch diese psychiatrische Modifikation mentale Autarkie abgesprochen. Suizid avanciert zu einer von vielen Arten, an einer Krankheit zu sterben, und kann dementsprechend verhindert werden, wenn rechtzeitig therapeutische Hilfe gesucht wird.

In Krellers Werk findet sich eine multiperspektivische Sichtweise auf den Suizidversuch der an einer schizophrenen Psychose erkrankten Figur, der Mutter namens Henrike. Sie betrachtet Suizid als den einzigen Weg, sich der eingebildeten Kontrolle zu entziehen. Die Repressivität des politischen Systems augmentiert die aufgetretene schizophrene Psychose. Henrikes Perspektivlosigkeit und besonders die ihrer Kinder Levin und Ole, die in vielzähligen Kommentaren expliziert wird, intensiviert die Erkrankung (Kreller 56). Ihr Suizidversuch ruft bei ihren Kindern Angst (Kreller 166, 180) und bei Levins Freundin Emma, der es trotz Dunkelheit und der Kälte des ätzenden Salzwassers gelungen ist, sie aus dem Meer zu retten, Wut hervor (Kreller 166-69). In ihrer chiastisch formulierten erlebten Rede offenbart Emma, dass sie die Selbsttötungsabsichten der psychisch kranken Frau durchschaut und auf diese mit ablehnender Vehemenz reagiert (Kreller 174). Emma zeigt Empathie für den benommenen Ole, indem sie den Selbstmordversuch seiner psychisch kranken Mutter als Unfall maskiert (Kreller 175). Die tiefe Dämmerung und die fröstelnde Kühle treten antithetisch dem milden, sonnendurchfluteten Tag entgegen, wodurch die innere Welt der, an einer schizophrenen Psychose erkrankten, Figur illustriert wird. Die Chronobiologie betont die Emotionsdarstellung.

Schließlich sind negativ konnotierte Katachresen zu nennen, die die pathologische Sicht der an Anorexie erkrankten Personen auf die Nahrungsaufnahme akzentuieren. So indiziert die kranke Figur Lucinda in unmittelbarer Darstellung im dramatischen Modus ihren Ekel, indem sie ihr Essen mit Würmern 
vergleicht. Diese Parallele verweist auf das Vanitas-Motiv der barocken Lyrik und deutet kataphorisch auf den Tod der Figur hin (Schützsack 137).

\subsection{Originelle Metaphern.}

Dem zweiten Typus sind Situationen zuzuordnen, in denen die Katachresen durch neue Metaphern ersetzt werden (Winko 334). Als ein Beispiel ist die Evokation positiver Emotionen im Zusammenhang mit Anorexie in dem Jugendroman Leute, ich fühle mich so leicht von Alexa Henning von Lange zu nennen. Die, an anorexia nervosa erkrankte, Figur Lelles ist stolz auf ihre Selbstdisziplin und vergleicht sich mit einem Genie, das bestimmte Dispositionen für das Hungern erfüllt (Henning von Lange 23). Eine ähnliche Einstellung der Erkrankten schildert Lara Schützsack in ihrem Werk Und auch so bitterkalt. Die krankheitsbedingt verzerrte Wahrnehmung der Zentralfigur wird durch die unsichere autodiegetische Erzählinstanz perspektiviert, deren Sichtweise durch das junge Alter und die mit ihm korrespondierende Naivität, Unerfahrenheit und euphorische Begeisterungsfähigkeit eingeschränkt ist. Die kindliche Figur Malina betrachtet die pathogene Transformation ihrer Schwester Lucinda als eine ästhetische Metamorphose und vergleicht sie mit einer Königin, die „ohne Angst dahin geht, wo es wehtut“ (Schützsack 146). Aus Malinas infantiler Perspektive erscheint der Tod ihrer Schwester ambivalent: Einerseits wird er aufgrund der von Lucinda forcierten Verklärung mit dem Elysium assoziiert. Andererseits stellt er eine unausweichliche Notwendigkeit dar, die humanistisch legitimiert und onomastisch verstärkt wird: „damit die anderen nicht geblendet werden“ (Schützsack 174). Auf die tödlichen Konsequenzen der Erkrankung verweist kataphorisch der Sturz des Stuckengels (Schützsack 23). Die Unausweichlichkeit des letalen Ausgangs wird auch durch die, von der Decke des Kinderzimmers abgefallenen und auf dem Fußboden verstreuten, Plastiksterne untermauert (Schützsack 169). Aus Lucindas Perspektive stellt der Tod eine Befreiung dar (Schützsack 39, 131, 147, 151). Die Krankheit macht sie zu einer Gefangenen ihrer selbst. Daher stellt sie sich vor, sie verwandle sich in eine Libelle, einen Vogel oder in Wasser (Schützsack 131). Lucinda träumt von einer eskapistischen Reise nach Tenebrien (Schützsack 10). In dem Namen des Landes lässt sich eine Allusion an den Topos der tenebrae erkennen. Dieser wird jedoch antithetisch verwendet: „Tenebrien ist das Land, in das alle gehen, die nicht für unsere Welt gemacht sind. Die Dünnhäutigen, die Gläsernen, diejenigen, die zu viel wünschen, diejenigen, die zu viel gewagt und zu viel verloren haben“" (Schützsack 10). Damit stilisiert sich die erkrankte Figur Lucindas zu einer Muse mit uner- 
schöpflicher Kraft, die verehrt, begehrt und bewundert wird und die sogar den Tod herbeiführen kann. Die Idee ihrer romantischen Verwandlung ergreift von der Figur Lucindas Besitz und infiziert in einer Kettenreaktion andere Figuren. Lucinda manipuliert diese und bewegt sie dazu, ihre Ideale von Romantik und Femininität und ihre verklärende Sichtweise sowohl auf die Erkrankung als auch auf den Tod einzunehmen (Schützsack 146). Der Stoizismus der Göttin Lucinda wird durch den Vergleich mit dem klaglosen Leiden des Hauses akzentuiert: „Armes altes Haus, denke ich, so krank, und wir dürfen dir nicht helfen. Keinen Ton gibst du von dir. Ob du irgendwann einfach stirbst?" (Schützsack 23). Diese Parallelisierung korreliert mit der Katachrese, die bei der Kreation von Krankheitsbildern häufig ihre Verwendung findet. Es handelt sich um die Sprachlosigkeit (Schützsack 163-164, Götz, Freytag, Kreller, Höfler, Vogel, Höfler, Tiefseequalle).

Eine ähnlich positive Sichtweise auf den Tod entwickelt die kindliche Figur Lucie aus Dita Zipfels Werk Wie der Wahnsinn mir die Welt erklärte. Sie betrachtet das plötzliche Verschwinden ihres älteren, an Demenz erkrankten Freundes als eine Verwandlung in einen Phönix (Zipfel 178). Diese Hoffnung versprechende Vorstellung überdeckt ihre Traurigkeit.

Die soziale Umgebung der an Anorexie erkrankten Figur Lucindas erlebt das eigene Scheitern und die damit verbundene Traurigkeit. Besonders die Figur der Mutter Isa ist vom eigenen Versagen in ihrer Rolle betroffen (Schützsack 163). Der Austragungsort der Konflikte zwischen den kontrastierenden Figuren der Mutter Isa und der Tochter Lucinda ist die Küche. Dieser Raum wird in der Psychoanalyse mit dem Mutterleib assoziiert (Funke, Psychoanalyse 104). Dementsprechend wird Isas Unvermögen als Mutter, die für die Versorgung ihrer Kinder mit den wichtigsten Nährstoffen verantwortlich ist, akzentuiert. Die Ängste der Figur Isas werden explizit thematisiert. Sie manifestieren sich aber auch implizit in Verboten, die an die jüngere Tochter Malina im Hinblick auf ihre Essgewohnheiten gerichtet werden (Schützsack 136) und auch in der Ablehnung der eigenen Selbstversorgung (Schützsack 163). Die paralysierende Angst erreicht ihren traurigen Höhepunkt in lähmender Apathie, gerade dann, als der Handlungsbedarf dringend und der, die Tochter Lucinda rettende, Notruf zu tätigen ist (Schützsack 173).

Die psychische Überforderung, die der Tod der besten Freundin hinterlässt, schildert Stefanie de Velasco in ihrem Roman Kein Teil der Welt. Der Verlust- 
schmerz wird mit kochender Himbeergrütze verglichen (de Velasco, Kein Teil der Welt 378). Hier findet eine Parallelisierung statt. Der Gemütszustand der jugendlichen Esther wird mittels der Metapher - vor Wut kochen - wiedergegeben. Sie empfindet diese starke Emotion gegenüber ihren eigenen Eltern, gegenüber der Mutter der unter mysteriösen Umständen getöteten Freundin, und gegenüber den Ältesten der Glaubensgemeinde der Zeugen Jehovas (de Velasco, Kein Teil der Welt 379-384). Diese Emotion expandiert auf die „Weltmenschen" (de Velasco, Kein Teil der Welt 380), indem ihre konventionalisierten Formeln der Emotionsdarstellung in Frage gestellt und als eine Simplifizierung diffamiert werden (de Velasco, Kein Teil der Welt 380).

Die Figur Bens aus dem Werk von Stefanie Höfler Der große schwarze Vogel führt die Emotion der Traurigkeit auf den Schatten, den der große schwarze Vogel auf ihn wirft, zurück (Höfler, Vogel 97, 105). Diese Metapher fungiert als ein Euphemismus für den Tod (Höfler, Vogel 96-97). Die Metamorphose des großen schwarzen Vogels zu einem kleinen Amselmännchen und der süße, schwere Herbstduft von reifen Äpfeln versinnbildlichen den Neuanfang für die Zentralfiguren (Höfler, Vogel 182).

\subsection{Unkonventionelle Situationen.}

Der dritte Typus der Emotionsdarstellung lässt sich in unkonventionellen Situationen identifizieren. Dabei werden typisierte Situationen, die kulturell als prototypische Emotionsauslöser gelten, modifiziert und durch ungewöhnliche Konstituenten erweitert (Winko 335).

Als ein Beispiel für eine solche Situation ist der Umgang der Schwestern Malina und Lucinda untereinander zu nennen (Schützsack). Es lässt sich eine emotionale Kurve nachzeichnen: Ihren Ausgangspunkt stellt eine, innige, körperliche Berührungen nicht ausschließende, schwesterliche Beziehung dar (Schützsack 5-10). Mit dem Fortschreiten der Krankheit bröckelt sie wie die Fassade des unter Denkmalschutz stehenden Domizils der Schwestern. So empfindet Malina Zweifel und Angst vor der Veränderung, als ihre Schwester Lucinda sie, ihre durch Anorexie verursachte, Lanugobehaarung berühren lässt. Die Angst korrespondiert mit dem Gefühl der radikalen Fremdheit (Leskovec 53), die sich bei dem Anblick des behaarten Rückens sowie der stark hervortretenden Rippen, die einem Käfig ähneln, einstellt (Schützsack 131). Lucinda zelebriert ihre Verwandlung und ihre Macht über den Hunger und den eigenen Körper. Der konsultierte Therapeut reduziert die Schwere der Erkrankung 
auf einen typischen, weiblichen Adoleszenzkonflikt und nivelliert durch seine Fehleinschätzung die berechtigten Sorgen der Familienangehörigen um ihre Tochter und Schwester: „Diese Mädchen sind gewissermaßen in ihrer eigenen Welt gefangen, in der Verpuppung vom Mädchen zur Frau" (Schützsack 141). Eine prä- und intertextuelle Verflechtung im Sinne einer expandierenden Äquivalenz (Schmid Mentale Ereignisse 201) lässt sich zu Lelle und ihrem behandelnden Arzt Schaffrat, der ebenfalls die für die junge Frau lebensbedrohliche Situation verkennt, identifizieren (Henning von Lange 7). Sie ironisiert in ihrer erlebten Rede den „Scharfsinn“ des, bereits onomastisch diskreditierten, Therapeuten. Diese Reaktion indiziert den Stolz der jungen Frau auf ihre Leistung, kontinuierlich zu hungern.

Die Metapher der Verpuppung als Inbegriff weiblicher Pubertät ist antiken Ursprungs und wird literarisch mit einer ambivalenten Konnotation tradiert: So konzipiert beispielsweise Johann Wolfgang von Goethe seine idealisierte, jungfräuliche Figur Mignon als Nymphe, deren Flügel noch nicht entfaltet sind (Goethe 540). Er verweist auf die religiöse Auffassung der Verwandlung der reinen, durch den vorzeitigen Tod vom sündhaften Erwachsenwerden und Erwachsenenleben geretteten Kindfrau in einen unsterblichen Geist (Wetzel 292-303). Der vorzeitige Tod der androgynen Mignon hält den Prozess der Verpuppung auf und rettet die Figur vor weiblicher Reife (Goethe 540). Auch Lewis Carroll konfrontiert seine Figur Alice mit einer Verpuppung, die durch die begonnenen physischen und psychischen Veränderungen verunsichert und irritiert ist. Die, von ihr konsultierte männliche, Raupe reagiert auf die bevorstehende, selbstverständliche Metamorphose dagegen mit Indifferenz (Carroll 50). In dem von Schützsack entworfenen Krankheitsnarrativ erweist sich diese Metapher für die Figur Lucindas als fatal (Schützsack 174).

Als ein weiteres Beispiel für diesen Typus ist die Idee der Familie anzuführen. Oft geht die Darstellung des Familienlebens der erkrankten Figuren in Kinder- und Jugendliteratur mit positiven Emotionen einher (Steinhöfel, Götz, Freytag, Höfler, Mucks). Die Erkrankten können die Sorge, die ihre Familienangehörigen für sie empfinden, jedoch anders wahrnehmen: So verwandelt sich zu Beginn der Narration die freundliche und liebevolle Figur Lucindas mit dem Fortschreiten der Anorexie in eine wütende Furie (Schützsack 38). Die Anorexie erscheint als ein Versuch der intrapsychischen und interpersonellen Selbstbehauptung. Dieser Versuch stellt eine Art der „Selbstreform am eigenen Körper" (Bruch 90) dar und äußert sich als ein erbitterter Kampf um Auto- 
nomie, Individuation sowie um die vollständige Selbstkontrolle. Dabei erfolgt eine rigorose Auflehnung gegen das utopische Schönheitsideal einer attraktiven Frau und eine Abwehr sexueller Wünsche (Bruch 90). Das angestrebte Ziel ist die androgyne Geschlechtslosigkeit, die sehr deutlich an Lucindas körperlicher Deformation demonstriert wird (Schützsack 131), wodurch die Metapher der Verpuppung aktualisiert wird. Die Abgrenzung von anderen, die nicht nach dem Perfektionsideal der an Anorexie Leidenden leben, führt zur Überhöhung der eigenen Persönlichkeit, zu Disharmonie, zu unkontrollierten Wutausbrüchen der Figur sowie zu rigiden Urteilen über die nicht betroffenen Personen. Die Zerstörung des inneren sicheren Seelenraums, der vor Schmerz und Verlust schützt, bewirkt die Verschiebung der Grenze des eigenen Selbstgefühls. Auch sind die gesunde Öffnung und Abgrenzung des Eigenraums stark beeinträchtigt. Bei den an Anorexie erkrankten Personen existiert die Ich-Grenze nicht (Funke, Psychoanalyse 122-29). Aus dieser Grenzenlosigkeit, die durch die „blindwütige Suche nach Identität und Selbstgefühl“ verstärkt wird (Bruch 16), resultieren eine Störung des Differenzierungs- und Ablösungsprozesses sowie eine Disbalance zwischen einem kommunikativen Familienleben und dem persönlichen Rückzug (Funke, Psychoanalyse 126). Es folgen gegenseitige Schuldzuweisungen der Familienmitglieder und der Machtkampf mit dem Sammeln von „Trophäen des Widerstandes“ (Schützsack 17, 26, 27, 136, 144 und Henning von Lange 23). Dieses Ungleichgewicht der Macht manifestiert sich in der Anorexie nicht nur in den Konflikten zwischen der Figur der Patientin und ihren Eltern, sondern in ihrem Kampf mit sich selbst und korreliert mit einem Gefühl der Überwachung, der Bestrafung und des Gefangenseins.

Lucindas Anorexie zwingt sie zu einer strengen Askese sowie zur existenzbedrohlichen Unterdrückung des Lebenserhaltungstriebs. Sie führt zu emotionaler Desorientierung der anfangs noch lebensfröhlichen und vor Energie strotzenden Figur sowie zu sukzessiver Erschöpfung, die in ihrem Tod einen tragischen Tiefpunkt erreicht (Schützsack 174). Auf der Ebene der histoire wird dieser Rückzug stark kontrastiert. Zu Beginn der Narration ist Lucindas Eigenraum offen: Sie hält sich gerne im Freien auf, sie geht auf andere Figuren zu und sie wirkt sehr präsent in der Schule, im Freundeskreis und in der Familie. Im Fortgang der Narration und somit mit dem Fortschreiten der Neurose vollzieht sich sukzessive die Schließung der Grenze zum Eigenraum (Funke, Psychoanalyse 127). Dem Verschließen von Türen, das Lucinda mit sich steigernder Häufigkeit vollzieht, kommt hierbei ein symbolischer Charakter zu (Schützsack 
15, Habermas 169). Die Schließung des Eigenraums kulminiert schließlich in der vollkommenen Abgrenzung und der daraus resultierenden, sozialen Isolation. Der Tod der Figur ihres Freundes Jarvis beschleunigt diese, für die Figur Lucindas fatale, Entwicklung (Schützsack 151). Die emotionale Metamorphose der Figur wird auch anhand der veränderten Wahrnehmung der Räume betont: Lucindas Kinderzimmer als Ort, in dem die jugendliche Figur ihre Kreativität auslebte, verwandelt sich mit dem Fortschreiten ihrer Erkrankung in ein Gefängnis, das die Figur nicht verlassen kann (Schützsack 170). Eine gleiche Entwicklung vollzieht sich mit den Möbeln: Das Bett wird aus einem positiv konnotierten, das Gefühl der Geborgenheit evozierenden Boot zu einem Ort der Trauer und Verzweiflung und schließlich zum Grab (Schützsack 15, 58, 146, 169, 170, 174 und auch de Velasco, Tigermilch 15). Die Transformation des Bettes aus dem Spielobjekt, das die Schwestern „sicher durch die Nacht bringt“ (Schützsack 15), deutet implizit auf den, in der antiken Mythologie etablierten, Symbolcharakter des Bootes bzw. des Schiffs als einem Transportmittel in das Totenreich hin (Butzer 368). Somit lässt sich das kindliche Spiel der Schwester und besonders die Nomination des Bettes als „Höhlenboot“ (Schützsack 15) als Antizipation des letalen Ausgangs von Lucindas voranschreitenden Erkrankung interpretieren.

Weitere Beispiele finden sich in den Adoleszenzromanen Für Niemand von Tobias Elsäßer und Das Gegenteil von Hasen von Anne Freytag. Der psychisch traumatisierte und an einer sozialen Phobie erkrankte Nidal verkehrt die Idee der liebevollen Familie in ihr Gegenteil, als er seine sexuelle Orientierung auf den viel zu liebevollen Umgang in der Familie zurückführt (Elsäßer 14). Sein pathologischer Selbsthass manifestiert sich in dem Wunsch, von seinem Vater "geschlagen, in den Bauch getreten, ja ausgepeitscht [zu werden], bis das Blut spritzt“ (Elsäßer 13). Freytags Figur Marlene Meller, die Akne hat, muss stets mit einem vorwurfsvollen „Du-hast-dich-nicht-geschminkt-Blick“ seitens ihrer Mutter rechnen (Freytag 283). Marlene kann zwar ihre roten Narben im Gesicht mit Maquillage verdecken, aber nicht die tiefen Wunden, die letztlich ihre Metamorphose in ein Monster bewirken (Kuzminykh 2021).

\subsection{Variation des emotionstypischen Verhaltens.}

Dem vierten Typus der Emotionsdarstellung sind Situationen zuzuordnen, die das emotionale Ausdrucksverhalten variieren (Winko 335). Es handelt sich

hierbei um Situationen, in denen die Manifestationsformen von Emotionen in 
der innertextlichen Wirklichkeit vom konventionellen Gebrauch differieren. Als ein Beispiel kann die überraschend verhaltene emotionale Reaktion des vierzehnjährigen Ben auf den plötzlichen Herzstillstand seiner jungen Mutter gelten. Bens Stoizismus wird sogar von seiner Tante thematisiert und bildet einen scharfen Kontrast zu dem Verhalten seines Vaters, der zunächst einer tiefen Depression verfällt und sich anschließend einer rasenden Wut hingibt (Höfler, Vogel 51, 82).

Ein weiteres Beispiel gibt Stefanie de Velasco, indem sie ihre siebzehnjährige Figur Esther während der Beerdigungsfeier ihrer besten Freundin Sulamith statt Trauer zu empfinden, an Werbesprüche denken lässt. Mit verfremdeten Werbebotschaften kommentierte die Verstorbene zeit ihres kurzen Lebens die strengen Regeln der Wachtturmgesellschaft und stellte somit ironisierend-sarkastisch die Fundamente des aufgezwungenen Glaubenssystems in Frage. Durch den mentalen Rückgriff auf Werbeslogans wird einerseits die Zuneigung zwischen den Figuren Sulamiths und Esthers betont, andererseits deutet die Zitation der Mottos in der erlebten Rede auf Esthers Emanzipationsprozess von der Sekte hin (de Velasco, Kein Teil der Welt 382).

Eine andere Beerdigungsfeier endet in einer Eskalation, als der Ehemann der verstorbenen jungen Frau diese mit der Pest vergleicht (Höfler, Vogel 163). Allerdings betont die Antithese den Effekt der Paradoxie: „Ja, die Pest. Sie war echt. Sie war der echteste Mensch, den ich kenne, und der lebendigste" (Höfler, Vogel 163). Die Redundanz und die Steigerung des Adjektivs echt charakterisieren den inneren Aufruhr der unter Schock stehenden Figur.

Anders als erwartet fallen die Emotionen der adipösen, diffamierten und diskriminierten Figur Nikos aus (Höfler, Tiefseequalle). Als autodiegetischer Erzähler gibt er einen Einblick in seine innere Welt und seine Emotionen, während er latenten und offenen Verbalinjurien ausgesetzt ist. Niko ist sich auch der, ihm hinter seinem Rücken aufoktroyierten, Etikettierungen bewusst, die er aber mit einer brillanten Eloquenz pariert (Höfler, Tiefseequalle 5, 14, 28, 29, $30,31,32,33,44,96,112)$. Er reflektiert über das scheinbare Mitleid der Lehrer und konfrontiert sie mit freundlicher, jedoch rigoroser und berechtigter Kritik, indem er seine Überlegungen zu ihren gutgemeinten Inklusionsversuchen formuliert (Höfler, Tiefseequalle 34, 100). Jedoch bewirken diese das Gegenteil. Damit wird eine scharfe Diskrepanz zwischen Inklusion und Exklusion signalisiert (Höfler, Tiefseequalle 113). Die multiperspektivische Anlage des Romans mit 
zwei autodiegetischen (von Niko und Sera) und mehreren intradiegetisch-homodiegetisch, intern fokalisierten Erzählstimmen (von Melinda, Marko und Leni) betont die Differenz zwischen Selbst- und Fremdwahrnehmung (Höfler, Tiefseequalle 141-42, 150, 153). Aus dieser Konzeption resultiert jedoch kein offener Deutungsraum, sondern eine Form repetitiv-normierenden Erzählens, kontrastiert mit der Weltanschauung der Wandelfigur Seras, deren Konversion und Empathie für den ausgestoßenen, adipösen Niko Transfereffekte erzielen soll.

\subsection{Naturbeschreibungs- und Jahreszeitentopoi.}

Die Korrelation von Natur und Psyche bildet den fünften Typus der Emotionsdarstellung (Winko 335). Die Naturbeschreibungs- und Jahreszeitentopoi dienen als Wahrnehmungs- und Deutungsfolie für Emotionen, deren Semantik etabliert und leicht identifizierbar ist. Der propositionale Gehalt dieser Topoi wird einerseits durch eine lange literarische Tradition determiniert, andererseits ergibt er sich aus der Rekurrenz auf das implizite Weltwissen. Dabei werden für die Darstellung von Emotionen sowohl Parallelisierung als auch Kontrastierung mit Naturphänomenen verwendet. Dies sei in Kürze exponiert: Als die Figur Esther mit der Nachricht vom Tod ihrer besten Freundin konfrontiert wird, verfällt sie in einen Schockzustand (de Velasco, Kein Teil der Welt 369). Diese emotionale Reaktion wird durch die Darstellung des langanhaltenden, eisigen und dunklen Winters versinnbildlicht. Dessen Kälte wird mehrfach betont, sie bildet eine Opposition zu den milderen Wintern der Vergangenheit. Die melancholische Traurigkeit wird nicht explizit thematisiert, sondern durch den Rückgriff auf den Jahreszeitentopos indiziert. Die Bewältigung des Schocks geht mit dem Einbruch des Frühlings am Ende der Narration einher. Dieser markiert den optimistischen Ausgang der Handlung (de Velasco, Kein Teil der Welt 428). Ähnliche ästhetische Verfahren finden sich bei Andreas Götz (237), Stefanie Höfler (Höfler, Vogel 148), Lilly Lindner (10) Lara Schützsack (5-10) um nur einige wenige Beispiele zu nennen.

Die tradierte Korrelation der jahreszeitlichen Naturphänomene kann jedoch aufgehoben, umgedeutet und umgewertet werden: So beschreibt Ben den Tag, an dem das Herz seiner Mutter aufgehört hat zu schlagen, als einen „strahlenden“ Tag (Höfler, Vogel 15). Das sonnendurchflutete Wetter steht in einem scharfen Kontrast zum Tod und markiert seine unzulängliche Radikalität (Leskovec 53). 
Auch die Zentralfigur in Tellers Adoleszenzroman setzt ihre Vorstellung vom Frühling in Opposition zu der etablierten Katachrese (Teller 122). Die Apathie gegenüber der Blütezeit unterstreicht ihre Depression, die in einen Suizid mündet.

\section{Fazit}

Als Fazit lässt sich festhalten, dass die Emotionsdarstellung von erkrankten Figuren in kinder- und jugendliterarischen Werken nuanciert ist. Emotionen werden sowohl explizit thematisiert als auch mit Hilfe des stilistischen Instrumentariums implizit indiziert. In Krankheitsnarrativen wird das gesamte Spektrum der menschlichen Emotionen ausdifferenziert, an dessen Spitze elementare Emotionen stehen. Diese werden durch komplexe Emotionen ergänzt, damit das Verstehen und das Nachempfinden der Figuren evoziert werden können. Nur auf diese Weise kann sich Empathie für diese höchst fragilen inneren Welten kindlicher und adoleszenter Figuren entwickeln.

\section{Literaturverzeichnis}

\section{Primärliteratur}

Best, Anne. Flippie-Bär ... für alle großen und kleinen Leute, die wissen wollen, wozu $A D(H) S$ gut ist! Langenkämper, 2005.

Buchholz, Katy. Meinst du, das hab ich mit Absicht getan? Wagner, 2007.

Buschendorf, Florian. Geil, das peinliche Photo stellen wir online. Verlag an der Ruhr, 2010.

Carroll, Lewis. Lewis Carroll The Complete Works. CRW, 2005.

De Velasco, Stefanie. Kein Teil der Welt. KiWi, 2020.

De Velasco, Stefanie. Tigermilch. KiWi, 2015.

Elsäßer, Tobias. Für niemand. Fischer, 2014.

Fontane, Theodor. Effi Briest. Aufbau Verlag, 2019.

Freytag, Anne. Das Gegenteil von Hasen. Heine, 2020.

Frisch, Max. Homo Faber. Suhrkamp, 1957.

Funke, Cornelia. Reckless. Steinernes Fleisch. Dressler, 2010.

Goethe, Johann Wolfgang. Wilhelm Meisters Lehrjahre. Reclam, 2008.

Götz, Andreas. Wir sind die Wahrheit. Dressler, 2020.

Habeck, Robert, und Paluch, Andrea. Zwei Wege in den Sommer. Sauerländer, 2007.

Hegemann, Helene. Axolotl Roadkill. Ullstein, 2010.

Hennig von Lange, Alexa. Leute, ich fühle mich leicht. cbt, 2008.

Herrmann, Elisabeth. Zartbittertod. cbt, 2020. 
Hesse, Hermann. Unterm Rad. Fischer, 1906.

Hoffmann, Heinrich. Struwwelpeter. Thienemann, 1844.

Höfler, Stefanie. Mein Sommer mit Mucks. Belz, 2015.

Höfler, Stefanie. Tanz der Tiefseequalle. Gulliver, 2018.

Höfler, Stefanie. Der große schwarze Vogel. Gulliver, 2019.

Huber, Lisa-Marie. Der Tod kriegt mich nicht: Mein Leben mit Leukämie. Arena, 2015.

Korschunow, Irina. Die Sache mit Christoph. dtv, 1988.

Kreller, Susan. Elektrische Fische. Carlsen, 2019.

Künzler-Behncke, Rosemarie. Der kleine Zappel-Felix. Ueberreuter, 2007.

Lindner, Lilly. Was fehlt, wenn ich verschwunden bin. Fischer, 2017.

Michaelis, Antonia. Der Märchenerzähler. Oetinger, 2011.

Moers, Walter. Prinzessin Insomnia. Knaus, 2018.

Nassauer, Max. Der gute Doktor. Braun\&Schneider, 1913.

Norden, Erich. Das kranke Hannchen. Perthes, 1891.

Novalis. Fragmente und Studien 1799-1800. Beck, 1981.

Pausewang, Gudrun. Die letzten Kinder von Schewenborn. Ravensburger, 1983.

Pausewang, Gudrun. Die Wolke. Ravensburger, 1987.

Pressler, Mirjam. Bitterschokolade. Beltz, 1980.

Richter-Peills, Charlotte. Magoria. Das Haus der Schatten. Rowohlt, 2013.

Rummel, Klaus, und Grütter, Thomas. Wie Zappelmax zu seinem Namen kam. Ein Buch der Lebenshilfe Osterholz. Langenbruch, 2001.

Schäfer, Ulrike. Tim Zippelzappel und Philipp Wippelwappel. Eine Geschichte für Kinder mit ADHS-Syndrom. Huber, 2003.

Schützsack, Lara. Und auch so bitterkalt. Fischer, 2016.

Spyri, Johanna. Heidi kann brauchen, was es gelernt hat. Perthes, 1881.

--. Heidis Lehr- und Wanderjahre. Perthes, 1881.

--. Wo Gritlis Kinder hingekommen sind. Perthes, 1883.

--. Am Felsensprung. Perthes, 1886.

Steinhöfel, Andreas. Rico, Oskar und das Herzgebreche. Carlsen, 2009.

--. Rico, Oskar und der Diebstahlstein. Carlsen, 2011.

--. Rico, Oskar und die Tieferschatten. Carlsen, 2008.

Strauß, Emil. Freund Hein: Eine Lebensgeschichte. Fischer, 1906.

Schulz, Almut. Rabenhaar. Erlebnisse eines ADHS-Kindes. BoD, 2006.

Shreve, Susan. Mein Freund Twist. Ein Junge hat ADS. Klopp, 2005.

Teller, Jane. Nichts. Was im Leben wichtig ist. dtv, 2010.

Tuckermann, Anja. Das verschluckte Lachen. Sauerländer, 2007.

Ury, Else. Nesthäkchen im Kinderheim. Meidinger, 1920.

von der Grün, Max. Vorstadtkrokodile. Bertelsmann, 1976.

Welsh, Renate. Drachenflügel. Obelisk, 1988. 
Zipfel, Dita. Wie der Wahnsinn mir die Welt erklärte. Hanser, 2020.

\section{Sekundärliteratur}

Alfels, Henrike. Literatur und Gefühl. Westdeutscher Verlag, 1995.

Balmer, Susanne. "Ganz ungewöhnlich eindrucksfähig. Krankheit in literarischen Weiblichkeitsentwürfen des 18. und 19. Jahrhunderts." Krank geschrieben: Gesundheit und Krankheit im Diskursfeld von Literatur, Geschlecht und Medizin. Rudolf Käser und Beate Schappach (Hg.), Scriptor, 2014, S. 45-62.

Blum, Mechthild, und Thomas Nesseler. Tabu Tod. Rombach, 1997.

Blümer, Agnes, Iris Schäfer, und Anika Ullmann. "Aktuelle Tendenzen zu Krankheit und Behinderung in Kinder- und Jugendliteratur und -medien." Kjløm, 66/3, 2014, S. 58-62.

Butzer, Gunter. Metzler Lexikon literarischer Symbole. Metzler, 2012.

Bruch, Hilde. Der goldene Käfig: Das Rätsel der Magersucht. Fischer, 1982.

Ehlenberger, Jan. Adoleszenz und Suizid in Schulromanen von Emil Strauß, Hermann Hesse, Bruno Wille und Friedrich Torberg. Peter Lang, 2006.

Ewers, Hans-Heino. Literatur für Kinder und Jugendliche. Fink, 2012.

Fenner, Dagmar. Suizid - Krankheitssyndrom oder Signatur der Freiheit? Eine medizinisch-ethische Untersuchung. Alber, 2008.

Frank, Arthur. The Wounded Storyteller. Body, Illness, and Ethics. Univ. of Chicago Press, 2013.

Funke, Dieter. Die dritte Haut. Psychoanalyse des Wohnens. Psychosozial Verlag, 2006.

Galling, Kurt. Die Religion in Geschichte und Gegenwart: Handwörterbuch in gemeinverständlicher Darstellung. Mohr, 2000.

Gebhardt, Lisette, und Klinski Michael. Nukleare Narrationen Kinder im Atomzeitalter - Berichte, Befunde, Bilder. EB-Verlag, 2019.

Gull, William. “Anorexia nervosa.” Lancet, 1, 1873, S. 516-17.

Habermas, Tillmann. Zur Geschichte der Magersucht. Eine medizinpsychologische Rekonstruktion. Fischer, 1994.

Herbert, Aron. Der Jugendsuizid in der Moderne. Universität Wien, 2014.

Holst, Nina, Schäfer, Iris, und Ullmann Annika. Narrating Disease and Deviance in Media for Children and Young Adults. Krankheits-und Abweichungsnarrative in kinder- und jugendliterarischen Medien. Peter Lang, 2016.

Jehle, Jörg-Martin. "Wie sieht die Verbrechenswirklichkeit aus und wie können wir Kriminalität begrenzen? Eine kriminologische Einführung." Das sogenannte Böse. Das Verbrechen aus internationaler Perspektive. Jörg-Martin Jehle (Hg.), nomos, 2020, S. 11-63.

Kuzminykh, Ksenia. “Die Genese des Bösen: philosophische und psychologische Dimensionen des Verbrechens als Narrativ im Jugendroman Das Gegenteil von Hasen von 
Anne Freytag", KiBUM, 2021, https://www.kibum-oldenburg.de/index.php?page=vidcasts\&vidcast_section=vidcasts_litwiss. Aufgerufen am 31. 08. 2021.

Kuzminykh, Ksenia. "Deviči obrazy v kontexte mirovoj literatury." New trends in slavistic studies. Suárez Cuadros (Hg.), urss, 2020, S. 527-37.

Mikota, Jana. "Magersucht in der populären Kinder- und Jugendliteratur". Narrating Disease and Deviance in Media for Children and Young Adults. Krankheits- und Abweichungsnarrative in kinder- und jugendliterarischen Medien, herausgegeben von Nina Holst, Iris Schäfer, und Annika Ullmann, Peter Lang, 2016, S. 73-90.

Mitterer, Nicola, und Wintersteiner, Werner. Wir sind die Seinen lachenden Munds. Der Tod - ein unsterblicher literarischer Topos. Studienverlag, 2010.

Lakoff, George, und Johnson, Mark. Leben in Metaphern. Konstruktion und Gebrauch von Sprachbildern. Carl-Auer, 1998.

Leskovec, Andrea. Einführung in die interkulturelle Literaturwissenschaft. Wissenschaftliche Buchgesellschaft, 2011.

Lexe, Heidi. "Der Tod und das Einmal-Eins." 1000 und 1 Buch, 4, 2004, S. 39-42.

Lexe, Heidi. "Down to the River. Das Wasser und der Tod." 1000 und 1 Buch, 1, 2009, S. 7-9.

Lüssi, Sara. “Zappel-Philipp und andere Fallgeschichten.” Krank geschrieben: Gesundheit und Krankheit im Diskursfeld von Literatur, Geschlecht und Medizin. Rudolf Käser und Beate Schappach (Hg.), Scriptor, 2014, S. 397-423.

Nickel, Sven. "Gesellschaftliche Reaktion auf Menschen mit (geistiger) Behinderung in der Kinder- und Jugendliteratur." Behindertenpädagogik, 4, 1999, S. 381-04.

Passante Elman, Julie. Chronic Youth. Disability, Sexuality, and U. S. Media Cultures of Rehabilitation. New York University Press, 2014.

Pitschke, Franziska. "Kranke Helden? Der Heldendiskurs im Kontext von Krebsnarrativen." Narrating Disease and Deviance in Media for Children and Young Adults. Krankheitsund Abweichungsnarrative in kinder- und jugenditerarischen Medien. Nina Holstet al. (Hg.), Peter Lang, 2016, S. 229-54.

Pope, Alexander. Essays on Criticism. Anodos books, 1970.

Rana, Marion. “Disability in Children's Literature: Tropes, Trends, and Themes." julit, 1, 2017, S. 26-45.

Reese, Ingeborg. Behinderung als Thema in der Kinder- und Jugendliteratur. Dr. Kovač, 2007.

Rüggemeier, Anne. "Graphic Illness. Die Krankheit im Leben der Geschwister am Beispiel von David B.s Comic Epileptic." julit, H. 1, 2017, S. 70-91.

Schäfer, Iris. Von der Hysterie zur Magersucht: Adoleszenz und Krankheit in Romanen und Erzählungen der Jahrhundert- und der Jahrtausendwende. Peter Lang, 2015.

Schäfer, Iris. Zur Ästhetik psychischer Krankheit in kinder- und jugendliterarischen Medien. Vandenhoeck und Rupprecht, 2020.

Schlichter, Anita. "Ein richtiger Indianer? Kranke Jungen in der Kinder- und Jugendliteratur." Gender and Disease in Literary and Medical Cultures. Anne-Julia Zwierlein und Iris Heif (Hg.), Winter, 2014, S. 213-34. 
Schmerheim, Philipp. "Narrative Verschiebungen: Wunder und das Spiel mit Perspektiven auf Behinderung und Inklusion." julit, 1, 2017, S. 46-68.

Schmid, Wolf. Mentale Ereignisse. de Gruyter, 2019.

Schmid, Wolf. Elemente der Narratologie. de Gruyter, 2005.

Stemmann, Anna. "Ich bin deshalb nicht schizo. Nur erwachsener, wenn es darauf ankommt Aspekte der gestörten Adoleszenz in Nils Mohls Es war einmal Indianerland.” Narrating Disease and Deviance in Media for Children and Young Adults. Krankheits- und Abweichungsnarrative in kinder- und jugendliterarischen Medien.Nina Holst at al. (Hg.), Peter Lang, 2016, S. 53-72.

Weiss, Simone. "Jugendliteratur über Pest, Aids und Krebs: Eine Bestandsaufnahme." Narrating Disease and Deviance in Media for Children and Young Adults. Krankheitsund Abweichungsnarrative in kinder- und jugendliterarischen Medien.Nina Holstet al. (Hg.), Peter Lang, 2016, S. 211-28.

von Glasenapp, Gabriele. "Simple Stories? Die Darstellung von Behinderung in der Kinderund Jugendliteratur." kjløm, H. 3, 2014, S. 3-15.

von Müller, Mareike, und Matthias Wermeling. "Geschichten ohne Ende: Präsentationsstrategien narrativierter Krankheitserfahrung." Diegesis, H. 2, 2016, S. 35-62.

Wetzel, Michael. Mignon. Die Kindsbraut als Phantasma der Goethezeit. Fink, 1999.

Winko, Simone. Kodierte Gefühle. Erich Schmidt, 2003. 


\title{
INNER WORLDS OF CHARACTERS WITH MENTAL ILLNESS IN CHILDREN'S AND YA LITERATURE
}

\begin{abstract}
Ksenia KUZMINYKH

Georg-August-Universität Göttingen, Germany

Humboldtallee 19, 37073 Göttingen

ksenia.kuzminykh@uni-goettingen.de
\end{abstract}

The paper discusses manifestations of emotions within illness narratives in children's and YA literature. It starts with a short historical and theoretical overview, which presupposes that emotions are intersubjective and culturally determined phenomena. The expression of emotions takes place on all linguistic levels, and can therefore be both explicit and implicit. The theoretical and historical overview is followed by a literary analysis of illness narratives and emotions of ill characters in children's and YA literature. Finally, by employing the research method of close reading, the article offers a typology of explicit and implicit emotions in the selected illness narratives for children and YA readers. The selection of texts is based on their exemplary character for the explication of emotions of the ill characters.

Keywords: children's literature, YA literature, explicit and implicit representations of emotion, illness narratives 


\title{
PRIKAZI UNUTARNJIH SVJETOVA KOD OBOLJELIH LIKOVA U DJEČJOJ KNJIŽEVNOSTI I KNJIŽEVNOSTI
} ZA MLADE

\author{
Sažetak \\ Ksenia KUZMINYKH \\ Georg-August-Universität Göttingen, Njemačka \\ Humboldtallee 19, 37073 Göttingen \\ ksenia.kuzminykh@uni-goettingen.de
}

Rad se bavi prikazivanjem emocija unutar narativa o bolesti u dječjoj književnosti i književnosti za mlade. Počinje kratkim povijesnim i teorijskim pregledom iz kojeg se izvodi pretpostavka da su emocije intersubjektivne i kulturološki uvjetovane pojave, kao i da se izražavanje emocija odvija na svim jezičnim razinama te da može biti eksplicitno i implicitno. Teorijski i povijesni pregled slijedi književna analiza pripovijesti o bolesti i emocija bolesnih likova u književnosti za djecu i mlade, nakon čega se u radu metodom pomnog čitanja donosi tipologija eksplicitnih i implicitnih emocija u odabranim narativima o bolesti u knjigama za djecu i mlade. Odabir tekstova temelji se na njihovoj podobnosti pri izražavanju emocija bolesnih likova.

Ključne riječi: književnost za djecu, književnost za mlade, eksplicitni i implicitini prikazi emocija, narativ o bolesti 